\title{
Functional consequences of the first reported mutations of the proto-oncogene PTTG1IP/PBF
}

\author{
W Imruetaicharoenchoke1,2,3, A Fletcher1,2, W Lu1,2, R J Watkins4, B Modasia1,2, \\ V L Poole1,2, H R Nieto1,2, R J Thompson'1,2, K Boelaert1,2, M L Read1,2, V E Smith1,2,* \\ and C J McCabe1,2,*
}

'Institute of Metabolism and Systems Research, University of Birmingham, Birmingham, UK ${ }^{2}$ Centre for Endocrinology, Diabetes and Metabolism, Birmingham Health Partners, Birmingham, UK 3Department of Surgery, Faculty of Medicine Siriraj Hospital, Mahidol University, Bangkok, Thailand ${ }^{4}$ Institute of Cancer and Genomic Sciences, University of Birmingham, Birmingham, UK *(V E Smith and C J McCabe contributed equally to this work)

Correspondence should be addressed to C J McCabe Email mccabcjz@bham.ac.uk

\begin{abstract}
Pituitary tumor-transforming gene 1-binding factor (PTTG1IP; PBF) is a multifunctional glycoprotein, which is overexpressed in a wide range of tumours, and significantly associated with poorer oncological outcomes, such as early tumour recurrence, distant metastasis, extramural vascular invasion and decreased disease-specific survival. PBF transforms NIH 3T3 fibroblasts and induces tumours in nude mice, while mice harbouring transgenic thyroidal PBF expression show hyperplasia and macrofollicular lesions. Our assumption that PBF becomes an oncogene purely through increased expression has been challenged by the recent report of mutations in PBF within the Catalogue of Somatic Mutations in Cancer (COSMIC) database. We therefore sought to determine whether the first $10 \mathrm{PBF}$ missense substitutions in human cancer might be oncogenic. Anisomycin half-life studies revealed that most mutations were associated with reduced protein stability compared to wild-type (WT) PBF. Proliferation assays narrowed our interest to two mutational events which significantly altered cell turnover: C51R and R140W. C51R was mainly confined to the endoplasmic reticulum while R140W was apparent in the Golgi apparatus. Both C51R and R140W lost the capacity to induce cellular migration and significantly reduced cell invasion. Colony formation and soft agar assays demonstrated that, in contrast to WT PBF, both mutants were unable to elicit significant colony formation or anchorage-independent growth. However, C51R and R140W retained the ability to repress radioiodide uptake, a functional hallmark of PBF. Our data reveal new insight into PBF function and confirm that, rather than being oncogenic, mutations in PBF are likely to be passenger effects, with overexpression of PBF the more important aetiological event in human cancer.
\end{abstract}
Key Words
- COSMIC
- TCGA
- PTTG1IP
- mutation
proliferation

http://erc.endocrinology-journals.org DOI: 10.1530/ERC-16-0340
๑) 2017 The authors Published by Bioscientifica Ltd. Printed in Great Britain

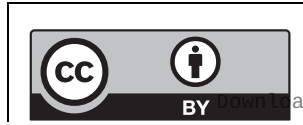

This work is licensed under a Creative Commons Attribution 3.0 Unported License.
Endocrine-Related Cancer (2017) 24, 459-474 


\section{Introduction}

Only a minority of mutations are likely to confer a specific growth advantage in vivo, resulting in clonal expansion and tumour development (driver mutations), whilst the majority of substitutions reported in sequencing databases such as The Cancer Genome Atlas (TCGA) and the Catalogue of Somatic Mutations in Cancer (COSMIC) are likely to be passenger mutations (Greenman et al. 2007). Functional clarification is now urgently needed to discern the driver mutations which may represent new or refined diagnostic, prognostic or therapeutic markers for particular types of cancer, and to more fully understand the mechanisms responsible for tumour formation and progression.

We previously characterised PBF (pituitary tumortransforming gene 1-binding factor; also known as PTTG1IP or c21orf3) as a proto-oncogene based on the transformation potential of $\mathrm{PBF}$ in vitro and its ability to induce tumours in xenograft models (Stratford et al. 2005). We and others have demonstrated that $P B F$ is overexpressed in pituitary, thyroid, breast and colorectal tumours, and that upregulation of the gene is significantly associated with poorer oncological outcome in a range of tumours (McCabe et al. 2003, Stratford et al. 2005, Watkins et al. 2010, Hsueh et al. 2013, Read et al. 2016). For instance, papillary thyroid cancer patients with high PBF expression at the time of diagnosis show decreased disease-specific survival compared to those who have low PBF expression (Hsueh et al. 2013). Colorectal tumours which show extramural vascular invasion have significantly higher PBF expression (Read et al. 2016), and high PBF promoter activity is associated with poorer clinical outcome and increased metastatic risk in breast cancer (Xiang et al. 2012). However, whilst transgenic PBF mice demonstrated increased AKT phosphorylation, thyroid gland enlargement, hyperplasia and macrofollicular lesions, they did not routinely develop tumours (Read et al. 2011). The role of PBF in tumourigenesis is therefore unclear.

$\mathrm{PBF}$ is ubiquitously expressed and highly conserved (Smith et al. 2011). It interacts with a range of proteins impacting on various cellular functions. The interaction with the human securin, PTTG, from which it gained its name, mediates the translocation of PTTG into the nucleus where it influences a number of cellular processes such as cell cycle regulation, genetic instability and gene transactivation (Vlotides et al. 2007). PBF also binds to the sodium iodide symporter (NIS) and monocarboxylate transporter 8 (MCT8), modulating their subcellular localisation, and regulating radioiodide uptake and thyroid hormone efflux, respectively (Smith et al. 2009, 2012, 2013, Read et al. 2011). Furthermore, overexpression of PBF increases p53 ubiquitination, resulting in dysregulated p53 cellular function (Read et al. 2014, 2016). Recent in silico efforts to identify driver genes amongst next generation sequencing data identified $P B F$ as a putative oncogene (Melloni et al. 2014).

Proto-oncogenes generally become oncogenic either through mutation or overexpression leading to gain-offunction. The TCGA (Chin et al. 2011, Tomczak et al. 2015) and COSMIC (Forbes et al. 2008, 2011, 2015) databases are now revealing mutational changes in protooncogenes that are considered to be oncogenic through enhanced expression, opening the possibility that these genes may, in rare cases, also act as oncogenes through functional activation. We utilised PBF as a paradigm of this idea, assessing whether the first 10 reported mutations of $\mathrm{PBF}$ in COSMIC might have oncogenic gain-of-function. Given that PBF is implicated in the aetiology of multiple tumour types, we determined the influence of the first 10 reported mutations of PBF upon cellular proliferation, before progressing a subset of mutations to canonical assays of cellular invasion, migration and anchorageindependent growth. Our data shed new light on PBF function and suggest that PBF upregulation, rather than mutation, is more critical to human tumour development and progression.

\section{Materials and methods}

\section{The Cancer Genome Atlas (TCGA)}

The TCGA project (http://cancergenome.nih.gov/) comprises a genomic data analysis pipeline that has resulted in the mapping of genomic alterations in more than 11,000 human tumours across 33 types of cancer (Chin et al. 2011, Tomczak et al. 2015). Data were obtained using the cBioPortal for Cancer Genomics (http://www. cbioportal.org/) (Cerami et al. 2012, Gao et al. 2013).

\section{Catalogue of Somatic Mutations in Cancer (COSMIC) database}

The COSMIC database (http://cancer.sanger.ac.uk/ cosmic), started in 2004, describes somatic mutation information in human cancers (Forbes et al. 2008, 2011, 2015). Data are collated through manual curation of scientific literature describing mutations in known cancer

Published by Bioscientifica Ltd. 
genes selected from the Cancer Gene Census (http:// cancer.sanger.ac.uk/cancergenome/projects/census/) and from large cancer genome datasets found in published literature and online data portals (Forbes et al. 2015). PBF mutation data were obtained from COSMIC in April 2013.

\section{Cell lines}

NIH 3T3 murine fibroblast and MCF7 breast cells were obtained from the American Type Culture Collection (ATCC) and maintained in DMEM (Sigma) and RPMI 1640 medium (Thermo Fisher Scientific), respectively. TPC1 and SW1736 thyroid carcinoma cell lines were kindly supplied by Dr Rebecca Schweppe (University of Colorado) and maintained in RPMI 1640 medium (Thermo Fisher Scientific). COS-7 African green monkey kidney epithelial and HeLa human cervical carcinoma cancer cell lines were acquired from the European Collection of Authenticated Cell Cultures (ECACC, Porton Down, UK) and maintained in DMEM, high glucose (Sigma). All were supplemented with $10 \%$ foetal bovine serum (FBS; Thermo Fisher Scientific), penicillin $\left(10^{5} \mathrm{U} / \mathrm{L}\right)$, and streptomycin $(100 \mathrm{mg} / \mathrm{L})$. As PBF is best characterised in thyroid and breast cancer, most of the experiments were performed using TPC1, SW1736 and MCF7 cells. Some experimental approaches required alternative or additional cell lines. For example, immunofluorescent determination of subcellular localisation was most effective in large, easily transfected cells such as HeLa cells and soft agar assays required stable, non-transformed NIH 3T3 cells.

\section{Plasmids and transfection}

Plasmids containing full-length PBF cDNA with or without a C-terminal haemagglutinin (HA) tag have previously been described (Stratford et al. 2005). All 10 PBF mutations were recapitulated in both HA-tagged and untagged PBF using the QuikChange II XL Site-Directed Mutagenesis Kit (Stratagene). FLAG-tagged PBF was generated through the ligation of an oligo encoding the FLAG sequence (DYKDDDK) into the pcDNA3.1+_PBF plasmid. The FLAG tag lies within the protein after residue E34. Previous attempts to tag PBF at the N-terminal end were unsuccessful due to the presence of a cleavable signal peptide; to overcome this, the FLAG epitope lies downstream of the cleavage site. The C51R and R140W mutations were also introduced into FLAG-tagged PBF. The NIS cDNA was housed in the pcDNA3.1+ vector with a C-terminal MYC tag (Smith et al. 2009). For stable transfections, untagged wild-type (WT) and PBF mutant cDNAs were cloned into the pCI-neo vector (Promega). Transfections were performed with TransIT-LT1 reagent (Geneflow, Lichfield, UK) following the manufacturer's protocol at a 3:1 reagent to DNA ratio and the experiments were performed after $24-48 \mathrm{~h}$.

\section{Immunofluorescence staining}

Immunofluorescence experiments were performed $48 \mathrm{~h}$ post-transfection in HeLa and MCF7 cells as described previously (Smith et al. 2009). Primary antibodies used included mouse monoclonal anti-HA.11 (1:200; BioLegend, San Diego, CA, USA), rabbit polyclonal anti-HA (Y-11) (Santa Cruz Biotechnology), mouse monoclonal anti-FLAG M2 (1:200; Sigma), rabbit polyclonal anti-PDI (1:200; Cell Signaling Technology), rabbit monoclonal anti-Golgin-97 (1:200; Cell Signaling Technology) and rabbit polyclonal anti-NIS ab104920 (1:500; Abcam). Cells were visualised using a Zeiss Axioplan fluorescent microscope (Zeiss) with a 100× objective.

\section{Western blotting}

Cells were harvested in radioimmunoprecipitation assay (RIPA) (50 mM Tris-HCl, pH 7.4, $150 \mathrm{mM} \mathrm{NaCl}, 1 \%$ vol/ vol IGEPAL CA-630, $6 \mathrm{mM}$ sodium deoxycholate, $1 \mathrm{mM}$ EDTA) with protease and phosphatase inhibitor cocktails (Sigma). Western blotting was performed as described previously (Smith et al. 2012). Proteins $(30 \mu \mathrm{g})$ were separated by SDS-PAGE using a 15\% acrylamide gel (12\% for the detection of NIS). Primary antibodies used were mouse monoclonal anti-HA.11 (1:1000; BioLegend), mouse monoclonal anti-FLAG M2 (1:500; Sigma), antiPBF antibody (made by Eurogentec (Seraing, Belgium) using the full-length PBF protein as an epitope), mouse monoclonal anti-MYC-Tag 9B11 (1:1000; Cell Signaling Technology) and mouse monoclonal $\beta$-actin AC-15 (1:10,000; Sigma).

\section{Half-life study}

MCF7 cells were transfected in 6-well plates with vector only (VO), HA-tagged WT and mutant PBF, and FLAGtagged WT and R140W PBF, and incubated for $48 \mathrm{~h}$. The cells were treated with $5 \mu \mathrm{L}$ anisomycin (Sigma) in $1 \mathrm{~mL}$ Opti-MEM serum-free media (Thermo Fisher Scientific) for 0,12 and $24 \mathrm{~h}$. Protein stability was determined through Western analysis. 


\section{Sorting Intolerant From Tolerant (SIFT) assessment}

SIFT (http://sift.jcvi.org/) is a software program that is used to predict whether an amino acid substitution impacts upon protein function. SIFT prediction is based on the degree of conservation of amino acid residues in closely related sequences collected through PositionSpecific Iterative Basic Local Alignment Search Tool (PSIBLAST) (Sim et al. 2012). An amino acid substitution that is predicted to have a phenotypic effect, with a SIFT score of less than 0.05 , is classified as 'damaging.' A mutation that is unlikely to have a phenotypic effect (SIFT score $>0.05$ ) is considered 'tolerated.'

\section{Glycosylation and dimerisation analysis}

For deglycosylating PNGase F treatment, $15 \mu$ g protein lysate from COS-7 transfected with either PBF-HA or PBF-N45/54A-HA was denatured for $30 \mathrm{~min}$ at $37^{\circ} \mathrm{C}$ with 10× Glycoprotein Denaturing Buffer (NEB, Ipswich, MA, USA) followed by incubation with $1 \mu \mathrm{L}$ PNGase $\mathrm{F}$ (NEB), $2 \mu \mathrm{L} 10 \% \mathrm{NP} 40$ and $2 \mu \mathrm{L} 10 \times \mathrm{G} 7$ buffer at $37^{\circ} \mathrm{C}$ overnight. Glycosylation status was determined through protein size discrimination by Western blotting. Cells were alternatively treated with the N-linked glycosylation inhibitor tunicamycin $(1 \mu \mathrm{g} / \mathrm{mL}$; Sigma) for $24 \mathrm{~h}$ prior to harvesting. The presence of a dimeric form of PBF $\sim 50 \mathrm{kDa}$ was determined through the omission of $\beta$-mercaptoethanol in the protein loading buffer. Dimerisation was also determined using proximity ligation assays (PLAs) which detect protein-protein interactions. HeLa cells were transfected with FLAG-PBF and PBF-HA, FLAG-PBF and VO, or PBF-HA and VO. After $48 \mathrm{~h}$ cells were fixed, permeabilised, blocked and incubated with primary antibody (mouse anti-FLAG and rabbit anti-HA) as performed in the immunofluorescent studies. The Duolink In Situ Kit (Olink, Uppsala, Sweden) was then used as per the manufacturer's instructions. Images were obtained using the Zeiss confocal LSM 510 microscope $\times 40$ objective.

\section{Cell proliferation assays}

For the bromodeoxyuridine (BrdU) assay TPC1 and MCF7 cells were transfected with $\mathrm{VO}$, untagged WT and mutant PBF. After $24 \mathrm{~h}$, cells were reseeded into 96-well plates with 8 replicates per condition. The Cell Proliferation ELISA, BrdU (colorimetric) kit (Roche) was used a further $24 \mathrm{~h}$ later following the manufacturer's protocol with a BrdU incubation time of $4 \mathrm{~h}$.
For the 3-(4,5-dimethylthiazol-2-yl)-5-(3-carboxyme thoxyphenyl)-2-(4-sulphophenyl)-2H-tetrazolium, inner salt (MTS) assay, $24 \mathrm{~h}$ post-transfection cells were reseeded into 96-well plates with 6 replicates per condition. The CellTiter 96 AQueous One Solution Cell Proliferation Assay (Promega) was used a further $24 \mathrm{~h}$ later following the manufacturer's protocol with an MTS reagent incubation time of $4 \mathrm{~h}$.

\section{D Boyden chamber invasion assays}

Forty-eight hours post-transfection with $\mathrm{VO}$, untagged WT PBF, C51R and R140W, TPC1 and MCF7 cells were switched to media containing 2\% FBS and were incubated for $16 \mathrm{~h}$. Cells were reseeded into Corning BioCoat Growth Factor Reduced Matrigel Invasion Chambers with $8.0 \mu \mathrm{m}$ polyethylene terephthalate (PET) membrane (Corning) and the chambers placed in 24-well plates with media containing 10\% FBS for a further $24 \mathrm{~h}$. Cells were then fixed in $95 \%$ ethanol and stained using Mayer's haematoxylin and eosin (Sigma). Images were captured using a light microscope with a $10 \times$ objective and the invading cells were counted with ImageJ software.

\section{Stable cell line generation}

TPC1 and NIH 3T3 cell lines were transfected with VO, untagged WT PBF and C51R and R140W housed within the pcDNA3.1+ and pCI-neo vectors, respectively. After $24 \mathrm{~h}$, transfected TPC1 and NIH 3T3 cells were selected with $1 \mathrm{mg} / \mathrm{mL}$ G418 (Geneticin). Single clones were isolated and expanded and the PBF expression levels of individual colonies were determined using Western blotting and TaqMan RT-PCR.

\section{Cell migration assays}

The classical scratch wound assays were performed in triplicate in 6-well plates with NIH 3T3 cells stably expressing VO, untagged WT PBF, C51R and R140W. Wounds were created with p10 pipette tips after the cells had reached $100 \%$ confluence. Images were captured from 3 different areas within each well at 0,4 and $6 \mathrm{~h}$. The area and percentage of wound closure were analysed using ImageJ software.

\section{Radioiodide uptake assays}

TPC1 and MCF7 cells were seeded into 24-well plates and transfected with VO, VO and NIS-MYC and NIS-MYC

Published by Bioscientifica Ltd 
with untagged WT PBF, C51R or R140W. Radioiodide uptake assays were performed $48 \mathrm{~h}$ after transfection as described previously (Smith et al. 2013). Briefly, NaI (final concentration $10 \mu \mathrm{M}$ ) and $0.1 \mu \mathrm{Ci}{ }^{125}$ I were added directly to the cell medium. After incubation at $37^{\circ} \mathrm{C}$ for $1 \mathrm{~h}$ medium was removed, and cells were washed rapidly with Hanks' balanced salt solution. Cells were lysed in $2 \%$ sodium dodecyl sulphate, and the radioactivity of the lysate was counted for $1 \mathrm{~min}$ in a gamma counter. Results are given as picomoles of $\mathrm{I}^{-}$per microgram of protein. To demonstrate specific uptake, the NIS inhibitor sodium perchlorate was used at $100 \mu \mathrm{M}$ to pretreat control wells for $1 \mathrm{~h}$ before the addition of radioiodide.

\section{Colony formation assays}

TPC1 cells stably expressing VO, untagged WT PBF, C51R and R140W were used in colony formation assays. The experiments were established in 6-well plates with appropriate cell density. After 14 days of incubation, colonies were stained with $0.005 \%$ crystal violet and the numbers of colonies were counted.

\section{Soft agar assays}

NIH 3T3 stably expressing VO, untagged WT PBF, C51R and R140W were used to determine transforming ability through soft agar assays. Cells were seeded into 6-well plates, with two layers of agar; the bottom layer consisted of $0.4 \%$ sterile agar, whilst the upper layer comprised a sterile $0.35 \%$ agarose and single-cell suspension. After 14 days of incubation, colonies were stained with $0.005 \%$ crystal violet and the numbers of colonies were counted.

\section{Statistical analyses}

Data were analysed using Student's $t$ test and MannWhitney $U$ test for comparison between two groups of parametric and nonparametric data, respectively. Analysis of variance and Kruskal-Wallis tests were used for betweengroup comparisons of multiple groups of parametric and nonparametric data, respectively. Significance was taken as $P<0.05$.

\section{Results}

\section{The genomic landscape of PBF in cancer}

Analysis of significant alterations to $P B F$ within the TCGA database, such as non-synonymous mutations, high-level amplification and homozygous deletion, revealed a relatively low frequency across the multiple datasets (Fig. 1A). Amplification events were most common, particularly in breast cancer patient xenografts, neuroendocrine prostate cancers and pancreatic cancers (Fig. 1A). The inclusion of low-level copy number events such as heterozygous loss and allelic gain demonstrated a broader spectrum of alteration frequency, with 14 tumour types having more than 50\% of tumours with an alteration in PBF copy number or mutation (Fig. 1B). Conversely, 6 tumour datasets had an alteration frequency of $<10 \%$ with a further 8 datasets having no alterations at all (Fig. 1B). Of the total number of alterations observed across all datasets, only 5\% were significant alterations whilst 54\% were heterozygous loss and $41 \%$ were allelic gain (Fig. 1B).

The first 10 PBF mutations reported via COSMIC occurred across all functional domains of PBF, with no obvious 'hot-spots' other than 3 substitutions at residue G106, within the transmembrane region (Fig. 1C). Two patients shared the same G106V substitution. The 8 sites of amino acid alteration are all highly conserved across various species (Fig. 1C). Mutations were apparent primarily in colorectal cancers $(N=8)$, but also in ovary (1), lung (1) and prostate (1) tumours (Table 1).

\section{Initial biochemical characterisation of the first 10 reported mutations of PBF}

We recapitulated all 10 mutations and examined the subcellular localisation of each mutant, HA-tagged at the C-terminus (green), within HeLa cells (Fig. 2A). HA-tagged R140W was difficult to detect through immunofluorescence (IF), so a version that was FLAGtagged at the N-terminal end was used and compared to FLAG-tagged WT PBF. Compared with untagged PBF, PBF-HA and FLAG-PBF demonstrated no significant difference in subcellular localisation (Supplementary Fig. 1, see section on supplementary data given at the end of this article). PBF is predominantly expressed in late endosomes and at the plasma membrane (Smith et al. 2009, 2012, 2013). Most mutants retained a WT vesicular intracellular localisation, apart from C51R, G106R and R140W (Fig. 2A). To investigate whether these cellularly expressed PBF mutants were stable in vitro, we carried out protein half-life studies in MCF7 cells transfected with HA-tagged WT and mutated constructs and treated with anisomycin to inhibit new protein synthesis for 0,12 and $24 \mathrm{~h}$ before harvesting. Broadly, all mutations were evident at the protein level and showed similar molecular

Published by Bioscientifica Ltd. 
A

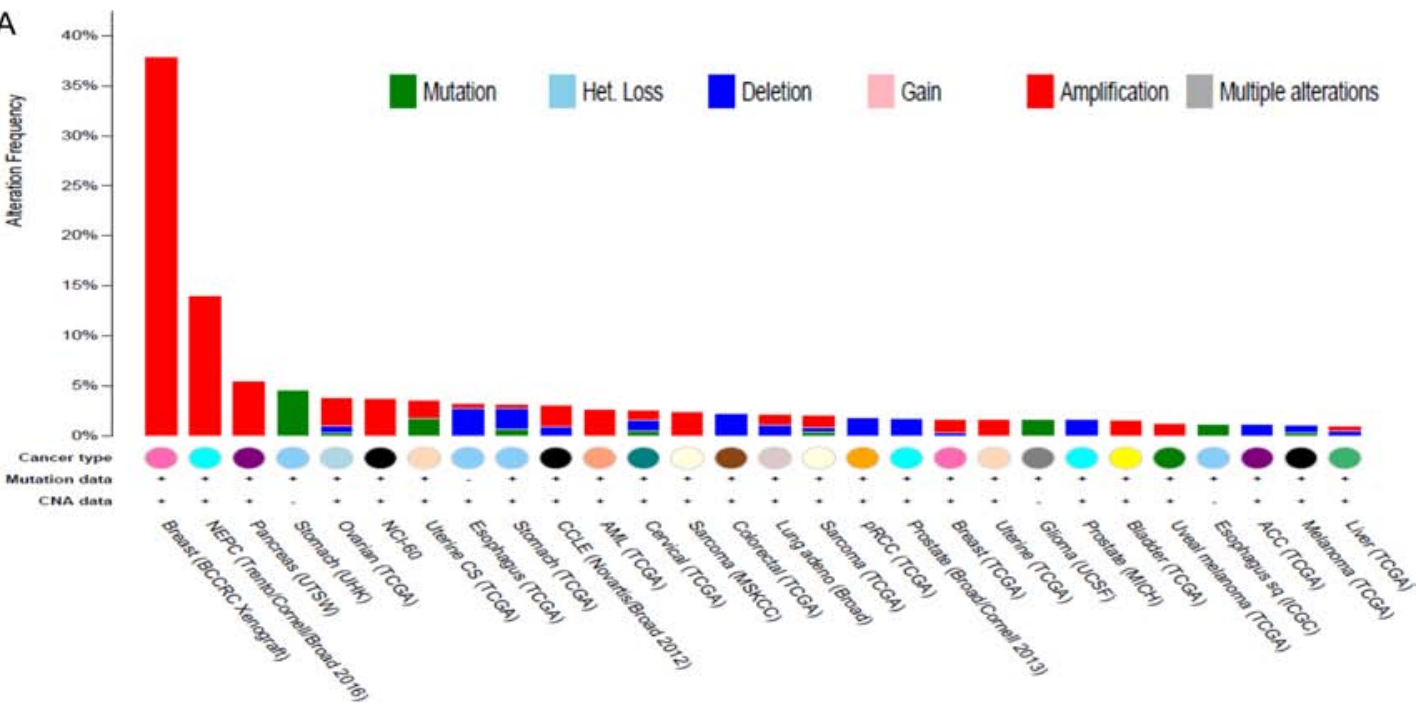

B

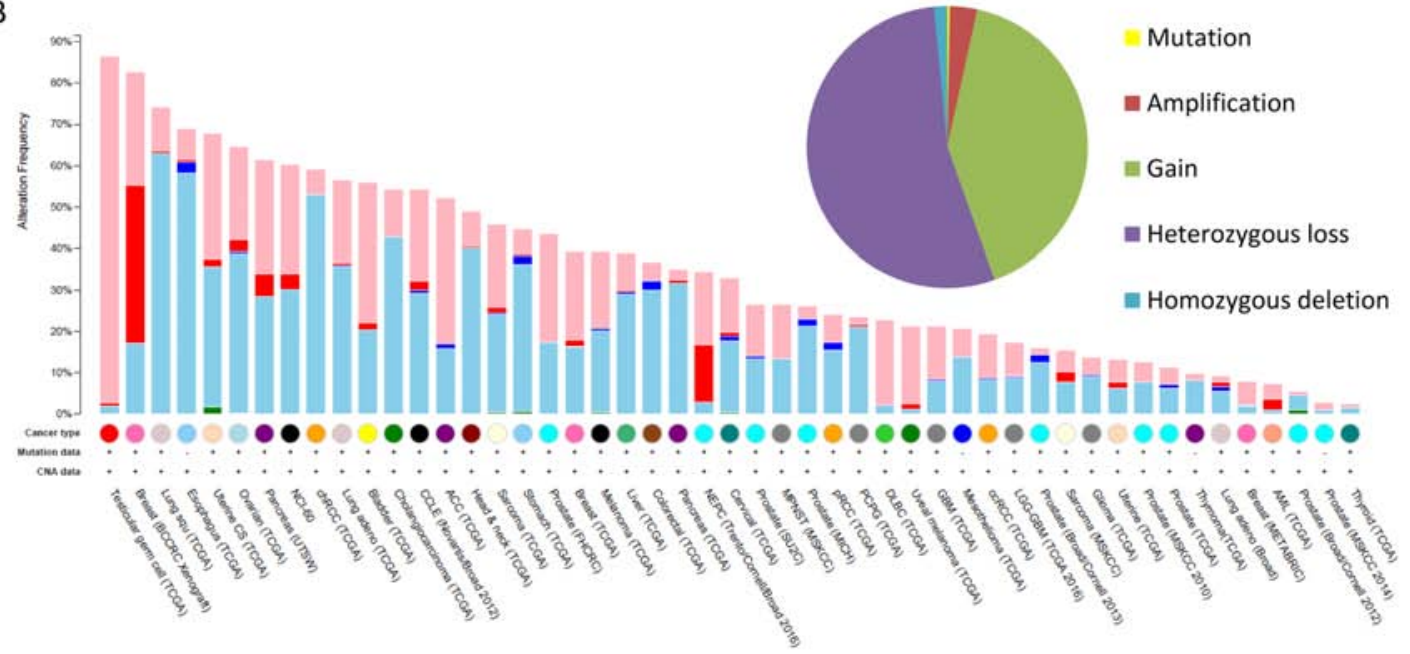

C

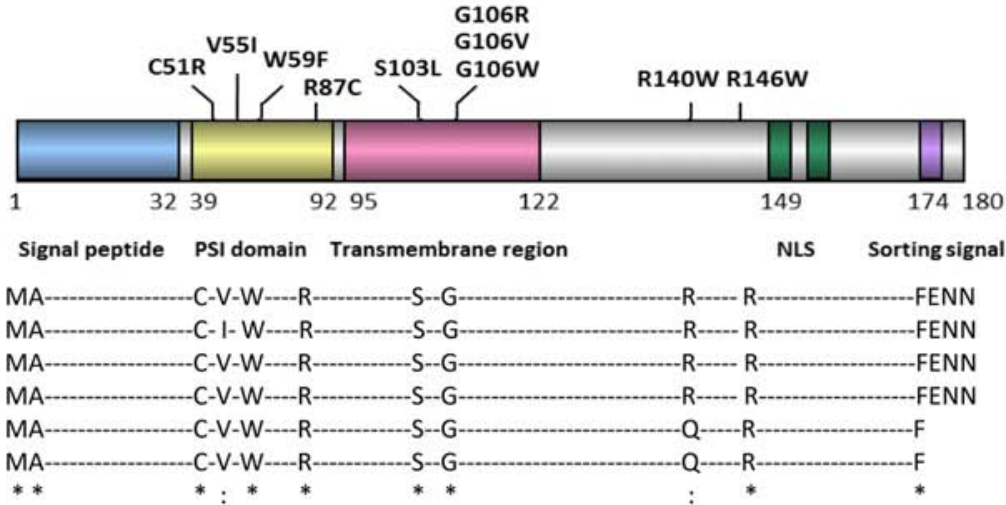

Figure 1

The genomic landscape of $P B F$ in cancer. (A) Alteration frequency $(>0 \%)$ of significant events in $P B F$, such as non-synonymous mutations, high-level amplification and homozygous deletion, in multiple human cancers in the TCGA database. (B) Alteration frequency of all events in $P B F$, including heterozygous loss and allelic gain, across all human cancer datasets with Copy Number Alteration information in the TCGA database. Inset - the distribution of all types of alteration to PBF. (C) Schematic diagram of PBF highlighting the first ten PBF mutations reported in the COSMIC database and their localisation within its functional domains (PSI, plexin-semaphorin-integrin; NLS, nuclear localisation signal). Below: amino acid conservation of the mutated residues across 6 animal species. *, identical/fully conserved amino acid; :, similar/conserved residues according to Clustal Omega analysis (http:// www.ebi.ac.uk/Tools/msa/clustalo/). 
Table 1 Clinical and histological data of patients with PBF mutations in various human cancers, according to the COSMIC database.

\begin{tabular}{|c|c|c|c|}
\hline PBF mutation & Gender & Age & Tumour origin \\
\hline C51R & $\mathrm{F}$ & 66 & Colon \\
\hline V55I & $M$ & 54 & Prostate \\
\hline W59F & $\mathrm{F}$ & 71 & Lung \\
\hline $\mathrm{R} 87 \mathrm{C}$ & $\mathrm{F}$ & 58 & Colon \\
\hline S103L & $M$ & 70 & Colon \\
\hline G106R & $\mathrm{F}$ & 63 & Ovary \\
\hline G106V1 & $M$ & 68 & Colon \\
\hline G106V2 & M & 45 & Colon \\
\hline G106W & $\mathrm{F}$ & 45 & Colon \\
\hline R140W & $\mathrm{F}$ & 58 & Colon \\
\hline R146W & $M$ & 78 & Colon \\
\hline
\end{tabular}

\begin{tabular}{|c|c|c|}
\hline Histology & TNM & $\begin{array}{l}\text { Vascular } \\
\text { invasion }\end{array}$ \\
\hline Adenocarcinoma, poorly diff. & T4bNOMO & - \\
\hline Carcinoma & Unknown & Unknown \\
\hline Adenocarcinoma, acinar type & T2aNOMO & Unknown \\
\hline Adenocarcinoma, moderately diff. & T3NOMO & - \\
\hline Adenocarcinoma, moderately diff. & T3N2aM0 & + \\
\hline Serous carcinoma & Unknown & Unknown \\
\hline Adenocarcinoma, moderately diff. & TxN1bM0 & - \\
\hline Adenocarcinoma, moderately diff. & T3NOMO & - \\
\hline Adenocarcinoma, well diff. & T3N0M0 & + \\
\hline Adenocarcinoma, moderately diff. & T3NOMO & - \\
\hline Adenocarcinoma, moderately to poorly diff. & T2NOMO & - \\
\hline
\end{tabular}

\begin{tabular}{l} 
Zygosity \\
\hline Heterozygous \\
Unknown \\
Unknown \\
Heterozygous \\
Heterozygous \\
Heterozygous \\
Heterozygous \\
Heterozygous \\
Heterozygous \\
Heterozygous \\
Heterozygous
\end{tabular}

F, female; $M$, male; $M$, metastasis; $N$, nodes; $T$, tumour

weights to WT PBF ( 30kDa; Fig. 2B). However, although detectable through IF, W59F frequently demonstrated low expression, and R140W could not be detected via C-terminal HA tagging. Scanning densitometry to determine the stability of PBF revealed that mutations C51R and V55I, both within the PSI domain of PBF, resulted in protein stability similar to WT, with a half-life of around $22 \mathrm{~h}$. FLAG-tagged R140W was also relatively stable compared to FLAG-WT PBF. In contrast, all other substitutions were markedly less stable than WT (Fig. 2C). Finally, SIFT assessment, based on sequence homology and physical properties of amino acids, demonstrated that all mutations except G106V were predicted to significantly alter protein function (Fig. 2D).

\section{Mutations of PBF influence post-translational processing}

WT PBF-HA and the majority of the mutants were largely detected as multiple bands between 25 and $37 \mathrm{kDa}$ (Fig. 2E). These bands were proposed to be differently glycosylated forms of PBF. Prediction software (http:// www.cbs.dtu.dk/services/NetNGlyc/) identified two putative sites of $\mathrm{N}$-linked glycosylation at residues N45 and N54. Tagging PBF either at the C-terminus with HA or near the N-terminus with FLAG was not predicted to result in different glycosylation patterns as determined via http://www.cbs.dtu.dk/services/ NetNGlyc/ (data not shown), although this was not confirmed experimentally. Following treatment of lysate from COS-7 cells transfected with PBF-HA with PNGase F to remove N-linked oligosaccharides, PBF was detected at its predicted molecular weight of $\sim 20 \mathrm{kDa}$ (Fig. 2F). Double substitution of both N45 and N54 with alanine also resulted in a protein of the same size (Fig. 2F), as did treatment with the glycosylation inhibitor tunicamycin (data not shown). Mutants C51R and G106R showed a banding pattern distinctive from all others, losing all but the lowest of the glycosylated bands at $\sim 25 \mathrm{kDa}$ (Fig. 2E). Discrete mutation of N45 and N54 suggested that these lower bands relate to a form of PBF glycosylated only at N45 whilst the upper bands represent protein modified at both sites (Fig. 2G). WT PBF does not appear to be glycosylated at N54 alone (Fig. 2G). Thus, mutation of C51R and G106R appears to prevent the full processing of this glycoprotein.

Our previous unpublished studies suggest that PBF can oligomerise. Analysis of these mutants highlighted a putative dimer at $\sim 50 \mathrm{kDa}$ (Fig. 2E). Under normal reducing conditions, the dimeric form of PBF is frequently difficult to detect. However, under non-reducing conditions a PBF-HA dimer is clearly present (Fig. 2H). A $50 \mathrm{kDa}$ band is also evident for the FLAG-tagged PBF protein (Fig. 2I), and PLAs clearly demonstrate an interaction between PBF-HA and FLAG-PBF (Fig. 2I) further confirming PBF dimerisation. The V55I, W59F, R87C and S103L mutations appeared to increase PBF dimerisation, which was evident even under normal reducing conditions (Fig. 2E).

\section{Mutations of PBF modulate cellular proliferation and subcellular localisation}

To date, PBF function has been studied most extensively in thyroid and breast cancer cell models (Stratford et al. 2005, Watkins et al. 2010, Xiang et al. 2012, Read et al. 2014). In vivo, PBF can enhance thyroid cell proliferation, as evidenced by gross thyroid enlargement accompanied by a significant induction of the proliferative marker cyclin D1 in transgenic mice (Read et al. 2011). 


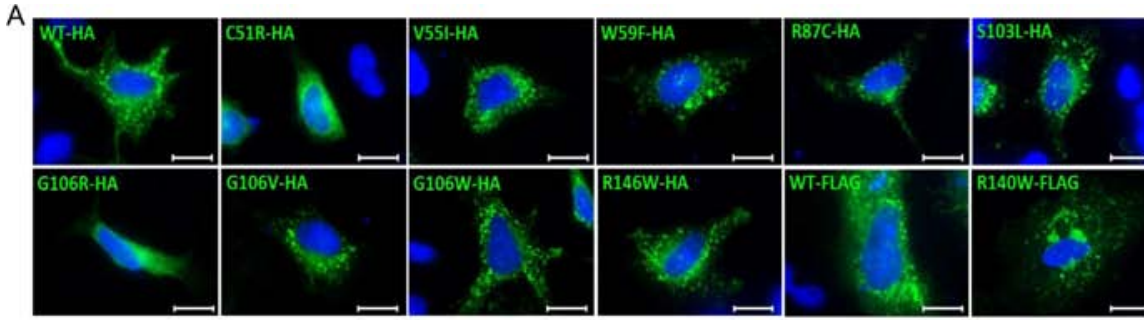

B

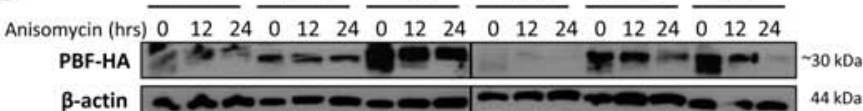

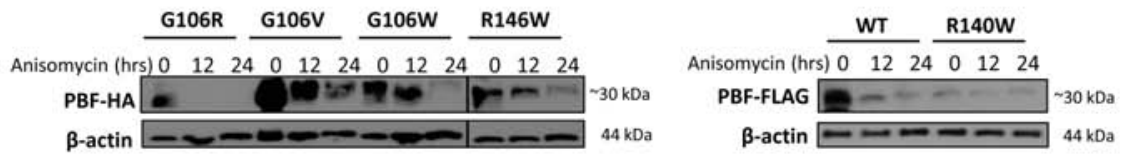

C $\quad 1.2$

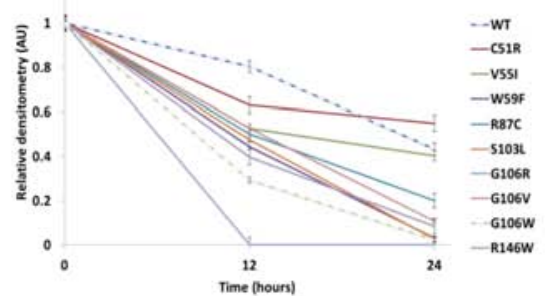

\begin{tabular}{|l|l|l|l|l|l|}
\hline Mutation & Position & Reference & Substitution & Prediction & SIFT Score \\
\hline
\end{tabular}

\begin{tabular}{|l|c|c|c|c|c|}
\hline CS1R & 51 & $\mathrm{C}$ & $\mathrm{R}$ & DAMAGING & 0 \\
V55I & 55 & $\mathrm{~V}$ & $\mathrm{I}$ & DAMAGING & 0.05 \\
W59F & 59 & $\mathrm{~W}$ & $\mathrm{~F}$ & DAMAGING & 0.02 \\
R87C & 87 & $\mathrm{R}$ & $\mathrm{C}$ & DAMAGING & 0 \\
S103L & 103 & $\mathrm{~S}$ & $\mathrm{~L}$ & DAMAGING & 0.01 \\
G106R & 106 & $\mathrm{G}$ & $\mathrm{R}$ & DAMAGING & 0.01 \\
G106V & 106 & $\mathrm{G}$ & $\mathrm{V}$ & TOLERATED & 0.12 \\
G106W & 106 & $\mathrm{G}$ & $\mathrm{W}$ & DAMAGING & 0 \\
R140W & 140 & $\mathrm{R}$ & $\mathrm{W}$ & DAMAGING & 0 \\
R146W & 146 & $\mathrm{R}$ & $\mathrm{W}$ & DAMAGING & 0 \\
\hline
\end{tabular}

00

E

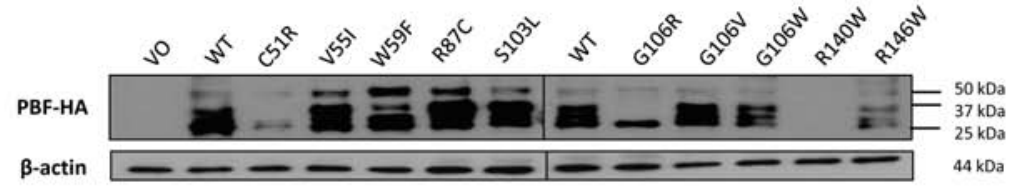

$\mathrm{F}$

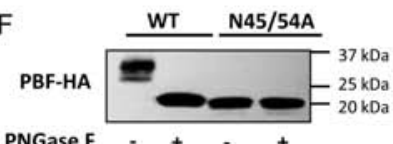

G

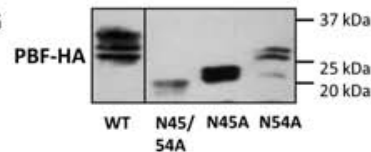

$\mathrm{H}$

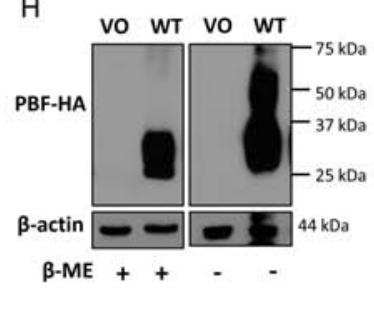

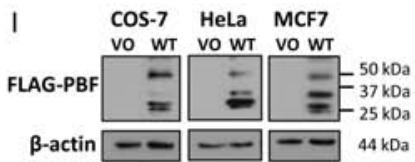

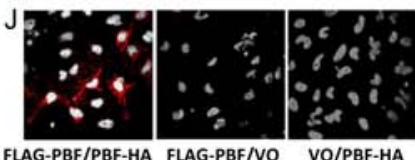

Figure 2

Expression, localisation and half-life of PBF mutations. (A) Subcellular localisation of each HA-tagged mutant (green) within HeLa cells using an anti-HA antibody. Blue indicates nuclear DAPI staining. As HA-tagged R140W is difficult to detect through immunofluorescent microscopy, FLAG-tagged wild-type (WT) and R140W PBF were stained using an anti-FLAG antibody. Magnification $=100 \times$. Bars $=20 \mu \mathrm{m}$. (B) Anisomycin half-life assays in MCF7 cells transfected with HA-tagged WT PBF and 9 mutations and treated for 0,12 or $24 \mathrm{~h}$, with quantification of relative PBF levels shown in Western blotting (FLAG-tagged WT and R140W are shown alongside). (C) Quantification of protein stability of the $\sim 25-37 \mathrm{kDa}$ isoforms of PBF in MCF7 cells following normalisation to $\beta$-actin expression. Data presented as mean values \pm s.E. $(n=2)$. (D) SIFT scores of all ten PBF mutations, where scores $<0.05$ are predicted to represent deleterious amino acid substitutions, which are defined as 'damaging.' (E) Western analysis of lysate from COS-7 cells transfected with vector only (VO), WT PBF and the 10 PBF mutants showing the expression levels and multiple forms of the HA-tagged PBF proteins. (F) PNGase F treatment of lysate from COS-7 cells transfected with WT PBF-HA results in a band at $\sim 20 \mathrm{kDa}$ and confirms N-linked glycosylation of PBF. PBF with a double substitution of N45 and N54 for alanine is also detected at $20 \mathrm{kDa}$ and cannot be modified by PNGase F confirming these are the only sites of glycosylation. (G) Western blotting of lysate from COS-7 cells transfected with HA-tagged PBF with discrete mutation of N45 and N54 suggests that both sites are glycosylated. $(\mathrm{H})$ Western analysis of WT PBF-HA expressed in HeLa cells using non-reducing (minus $\beta$-mercaptoethanol ( $\beta$-ME)) conditions clearly reveals the presence of a dimer $\sim 50 \mathrm{kDa}$. (I) Western blotting demonstrating the presence of the $\sim 50 \mathrm{kDa}$ band, and therefore the ability of FLAG-PBF to form dimers, in COS-7, HeLa and MCF7 cells transfected with WT FLAG-PBF and VO control. (J) Proximity ligation assays (PLAs) demonstrating an interaction between FLAG-PBF and PBF-HA in HeLa cells (red spots indicate $<30-40 \mathrm{~nm}$ distance between the anti-FLAG and anti-HA antibody epitopes and signify protein-protein interaction). VO co-transfections with either FLAG-PBF or PBF-HA represent negative controls.

http://erc.endocrinology-journals.org DOI: 10.1530/ERC-16-0340
C) 2017 The authors Printed in Great Britain
Published by Bioscientifica Ltd. 
We therefore sought to identify whether any of the described mutations conferred a growth advantage. Given that the G106 residue carried 3 mutations (G106R, G106V and G106W), initial proliferation assays were carried out in thyroid cells transfected with WT PBF and the G106 mutants. However, all 3 mutations failed to significantly alter proliferative capacity in both SW1736 (Fig. 3A) and TPC1 cells (Fig. 3B) using MTS assays and also in BrdU incorporation experiments (data not shown). Hence residue G106 was subsequently considered not to be a hotspot of mutational activation. Preliminary screening of all 10 mutations (data not shown) also enabled us to narrow our search to 2 mutations which increased cell turnover (C51R and V55I), and 2 mutations which repressed cell turnover (R140W and R146W) compared to WT. Thus BrdU assays, which indirectly assess DNA replication as a marker of cell division, revealed that C51R and V55I induced cell proliferation in TPC1 thyroid and MCF7 breast cells compared with WT, whilst R140W and R146W lost the pro-proliferative capacity of WT PBF (Fig. 3C and D). MTS assays, which act as a marker of cell number through indirectly measuring mitochondrial activity, revealed largely consistent findings, particularly with regard to the reduced proliferative influence of R140W (Fig. 3E and F) compared to WT PBF. For subsequent investigations we therefore confined ourselves to the 2 mutations which (i) showed the most obvious differences in cellular proliferation compared with WT PBF, (ii) were potentially damaging to protein function via SIFT, (iii) were apparent in the same tumour category (Table 1), (iv) showed altered subcellular distribution, (v) occurred at evolutionarily conserved sites, and (vi) were clearly expressed in vitro; C51R (N-terminal, within the PSI domain) and R140W (C-terminal, adjacent to the nuclear localisation signal). We next categorised the subcellular localisation of FLAGtagged mutants C51R and R140W more specifically through immunofluorescent microscopy in HeLa (Fig. 3G) and MCF7 (Fig. 3H) cells. C51R was mainly localised to the endoplasmic reticulum (ER), as evidenced by its co-localisation with the ER marker PDI. R140W, by contrast, was predominantly apparent in the Golgi apparatus, where it was co-located with the Golgi marker, golgin-97 (Fig. 3G and H). Thus we selected 2 candidate PBF mutations to explore in more functional depth.

\section{The influence of C51R and R140W mutants on cell invasion and migration}

Previous studies from our group revealed that PBF significantly induces cellular invasion and migration in breast cancer (Watkins et al. 2010). More recent investigations have demonstrated that PBF can induce invasion and migration in multiple cancer cells including thyroid and colorectal cells (Watkins et al. 2016). Furthermore, these studies suggest that this occurs at least partly through interaction with cortactin (CTTN), a scaffold protein that acts predominantly at the cell periphery to promote actin polymerisation and can thus exert a potent influence upon cell movement and invasion (Watkins et al. 2016). Here, the impact of the PBF substitutions on cell invasion was determined through 2D Boyden chamber cell invasion experiments in TPC1 and MCF7 cells transfected with VO, WT PBF, C51R and R140W mutants. As before (Watkins et al. 2010), WT $\mathrm{PBF}$ was markedly pro-invasive compared to $\mathrm{VO}$, in both TPC1 (Fig. 4A) and MCF7 (Fig. 4B) cells. However, both C51R and R140W substitutions entirely lost their invasive capacity, and in fact significantly repressed cellular invasion compared with VO control cells (Fig. 4A and B).

As cellular invasion is associated with and encompasses cell migration, we utilised classical scratch wound healing assays to measure cell migration in NIH $3 \mathrm{~T} 3$ cells stably transfected with VO, WT PBF, C51R and R140W. WT PBF had a significant pro-migratory capacity compared to VO controls (Fig. 4C and D). In contrast, both mutants C51R and R140W lost the ability to induce wound healing compared to stable WT cells (Fig. 4C and D). Therefore, 2 mutant forms of PBF discovered in patients with cancer, one N-terminal and the other C-terminal, both lost the ability to induce cell invasion and cell migration.

\section{PBF mutations retain the capability to repress radioiodide uptake in vitro}

The best-characterised function of PBF lies in its repression of radioiodide uptake via the cellular redistribution of the sodium iodide symporter, NIS (Boelaert et al. 2007, Smith et al. 2009, 2013, Read et al. 2011). To further examine the impact of the PBF C51R and R140W mutations, we conducted radioiodide (125I) uptake assays in TPC1 and MCF7 cells co-transfected with NIS-MYC and VO, WT PBF, C51R or R140W. Transient NIS transfection induced ${ }^{125}$ I uptake compared to VO (Fig. 5A and B). Co-transfection with WT PBF resulted in significantly reduced uptake in both TPC1 and MCF7 cells. Radioiodide uptake was higher in MCF7 than TPC1 cells, presumably reflecting differences in transient transfection efficiencies. Interestingly, both PBF mutants retained the ability to repress radioiodide uptake in both cell lines (Fig. 5A and B).

Published by Bioscientifica Ltd. 
A

SW1738

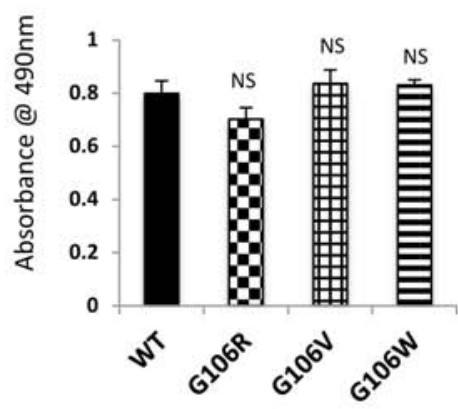

C

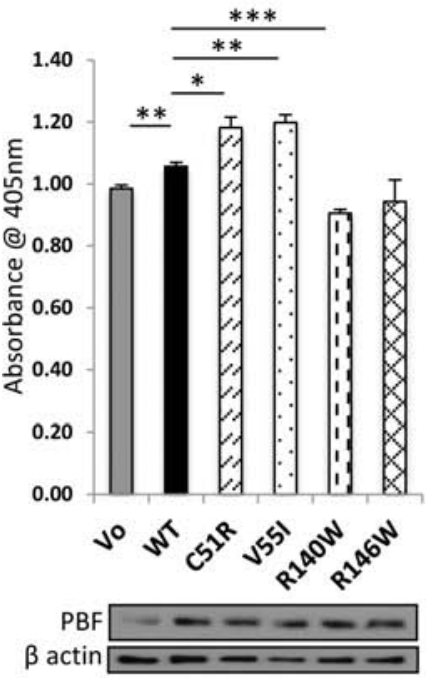

D

MCF7

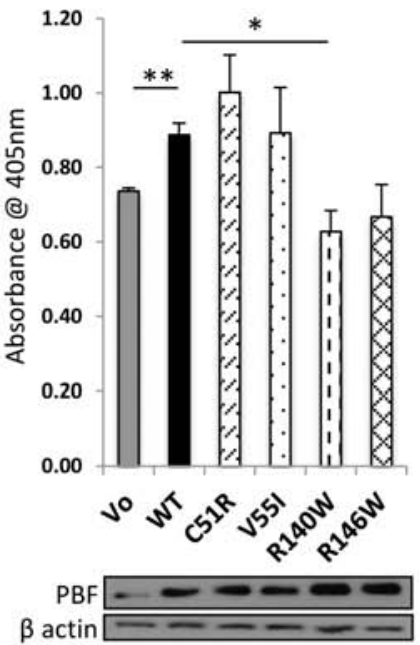

B

TPC1

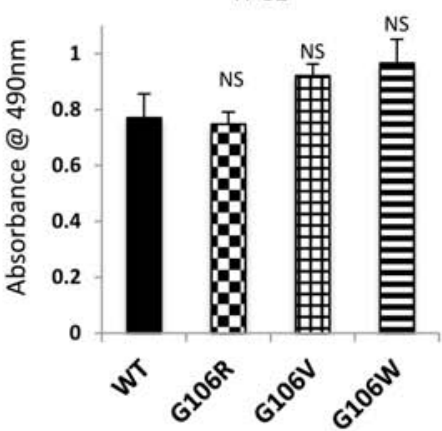

E

F

MCF7
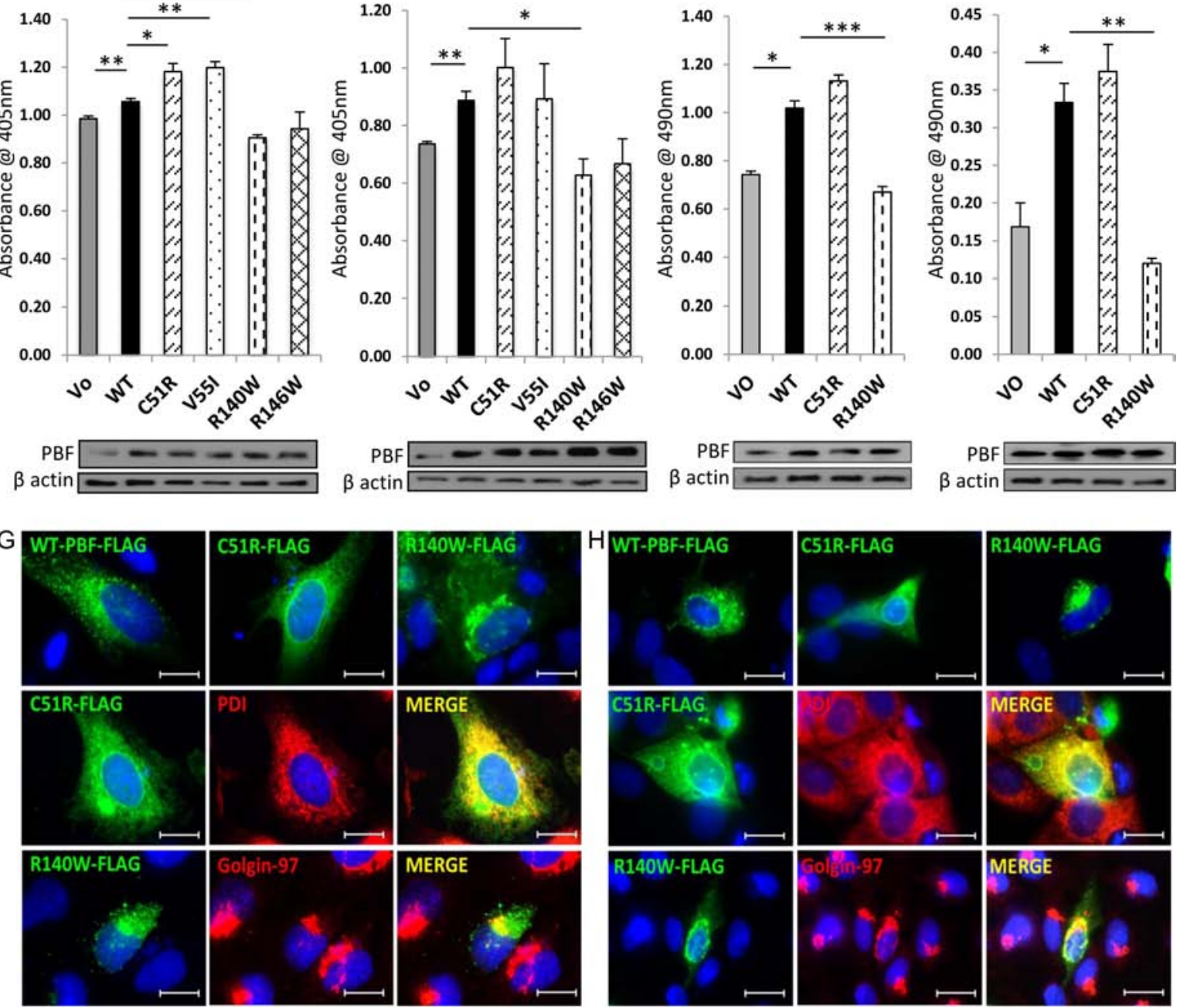

Figure 3

The influence of PBF mutations on thyroid and breast cell proliferation. MTS proliferation assays in SW1736 (A) and TPC1 (B) thyroid cancer cells demonstrated that 3 substitution mutations at residue G106 failed to significantly alter mitochondrial activity, compared with WT PBF, as a marker of cell number. BrdU cellular proliferation experiments in TPC1 (C) and MCF7 (D) cells transfected with VO, WT PBF, C51R, V55I, R140W and R146W mutants. MTS proliferation assays in TPC1 (E) and MCF7 (F) cells transfected with VO, WT PBF, C51R and R140W substitutions. Beneath - Western blots probed with anti-PBF antibody and $\beta$-actin as loading control demonstrate PBF expression to assess transfection. Data presented as mean values \pm s.E. $(n=3)$. $* P<0.05, * * P<0.01, * * * P<0.001$. Representative immunofluorescent microscopy of FLAG-tagged PBF and C51R and R140W mutants (green) and ER marker (anti-PDI Ab, red) or Golgi marker (anti-golgin-97 Ab, red) with subcellular co-localisation (yellow) in HeLa (G) and MCF7 (H) cells. Magnification $=100 \times$. Bars $=20 \mu \mathrm{m}$. 
A

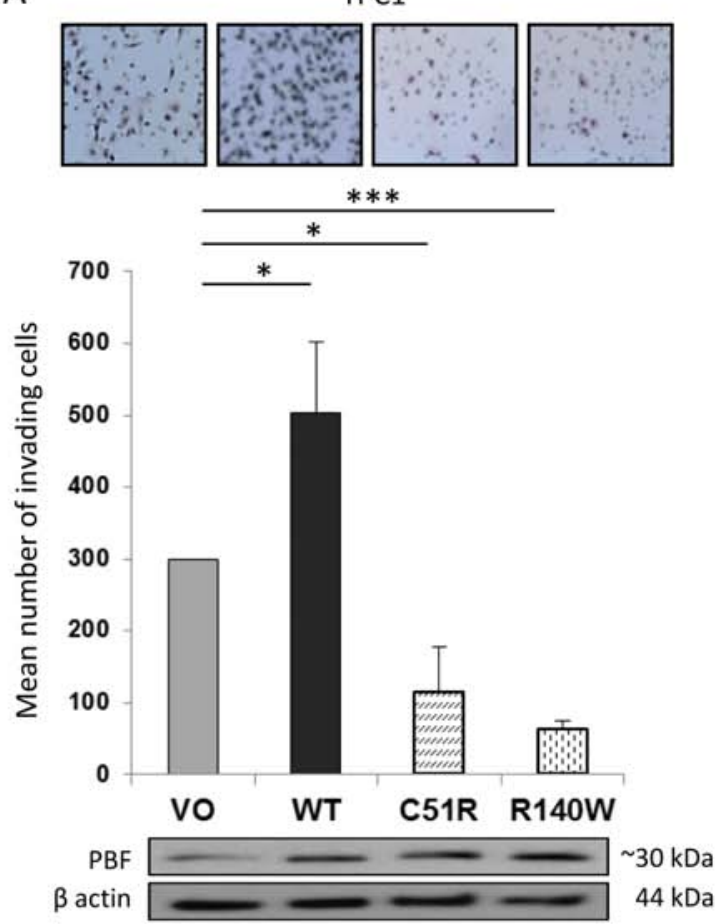

C

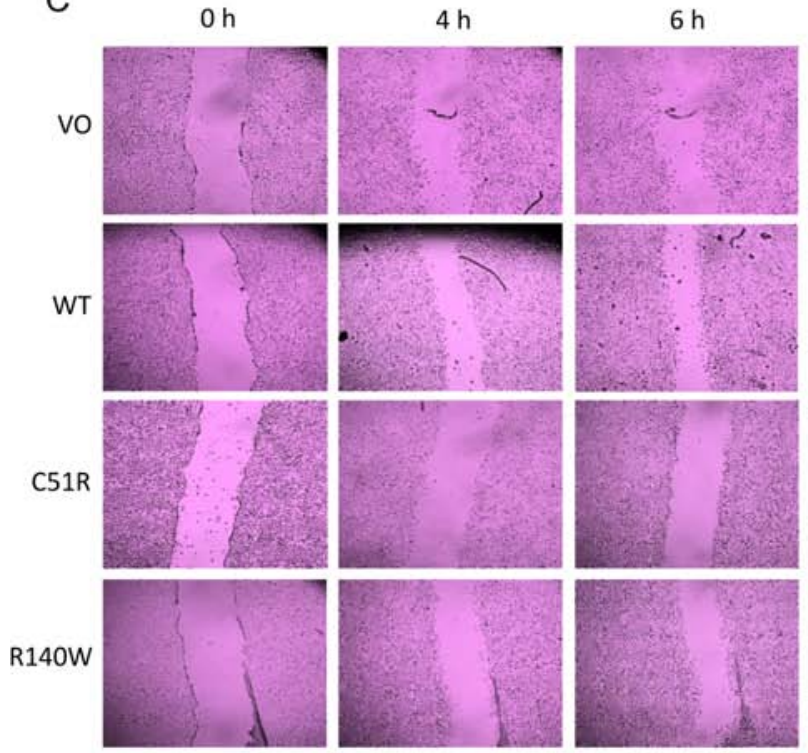

B

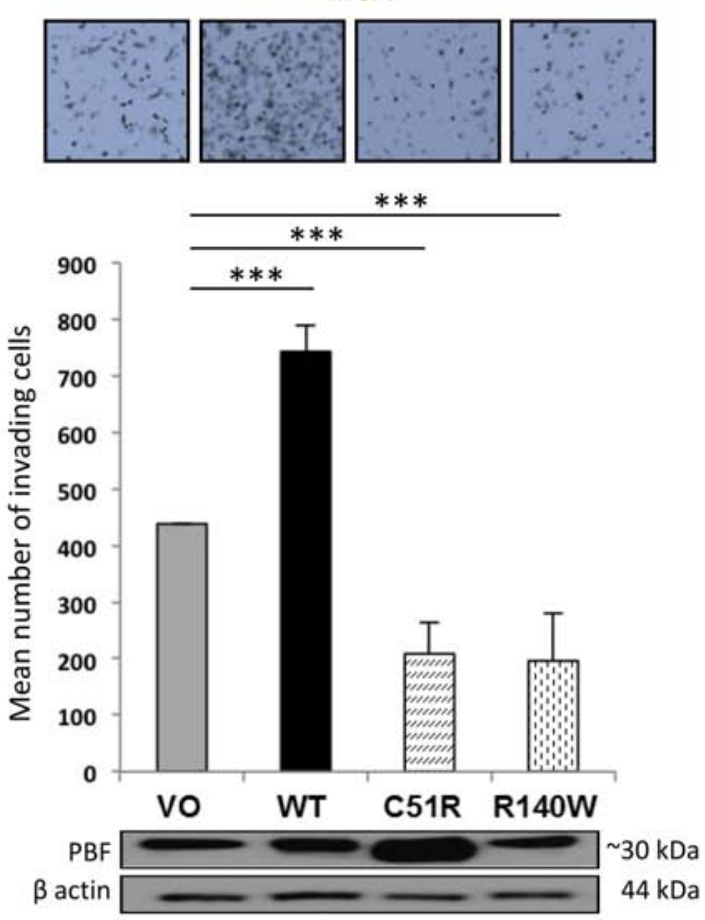

D VO WT C51RR140W

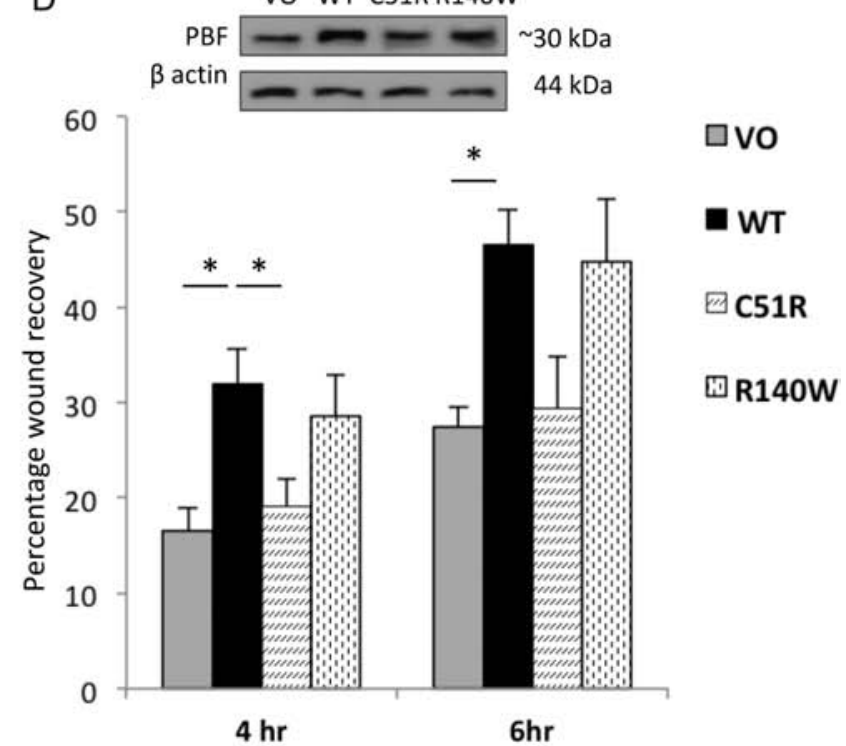

\section{Figure 4}

Invasive and migratory properties of C51R and R140W. 2D Boyden assay chamber cell invasion experiments in TPC1 (A) and MCF7 (B) cells transfected with VO, WT PBF, C51R and R140W mutants, with representative photomicrographs shown above. Data presented as mean values \pm S.E. $(n=2)$. (C) Representative classical scratch wound assays in NIH 3T3 cells stably transfected with VO, WT PBF, C51R and R140W substitutions at 4 and 6 h, with percentages of wound recovery shown graphically in D. Inset - Western blotting analysis of PBF levels in the stable NIH 3 T3 cells using an anti-PBF antibody. Data presented as mean values \pm S.E. $(n=3)$. ${ }^{*} P<0.05, * * * P<0.001$.

To examine this more closely, we determined the co-localisation of FLAG-tagged WT or mutated PBF with NIS-MYC through immunofluorescent microscopy in HeLa (Fig. 5C) and MCF7 cells (Fig. 5D). WT PBF co-localised with NIS predominantly in cellular vesicles, as we have reported previously (Smith et al. 2009, 2013). However, C51R and NIS mostly co-localised in the ER, and major subcellular co-localisation between R140W 
and NIS was apparent in the Golgi apparatus (Fig. 5C and D). Hence, although mutations C51R and R140W show altered subcellular localisation, they retain NIS

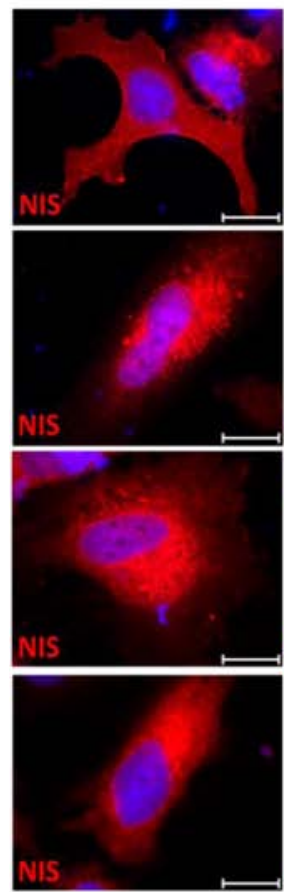

A

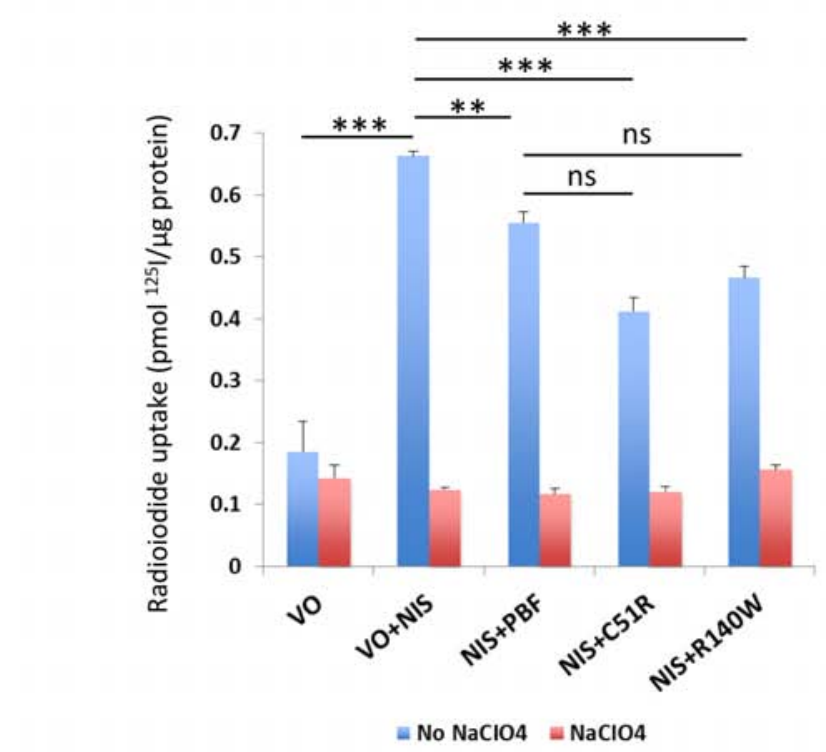

C

TPC1

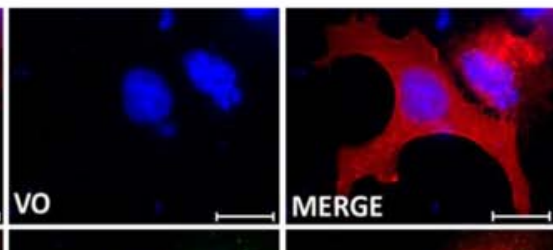

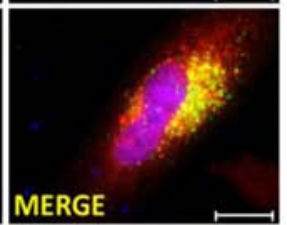
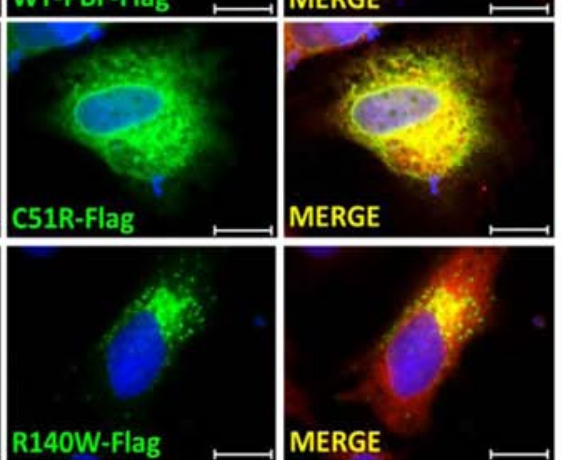

co-localisation and preserve the ability to repress radioiodide uptake. Furthermore, these data suggest for the first time that the ability of PBF to bind NIS

B

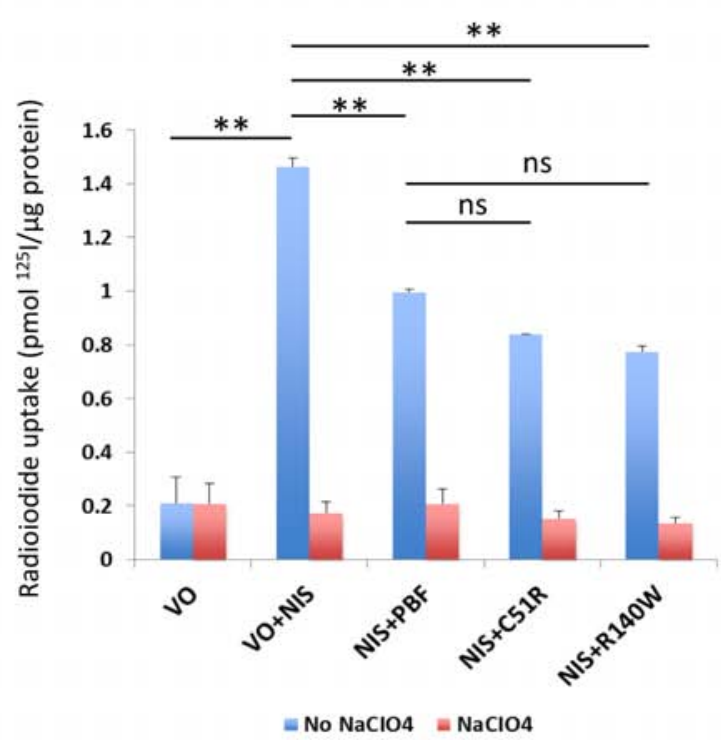

D

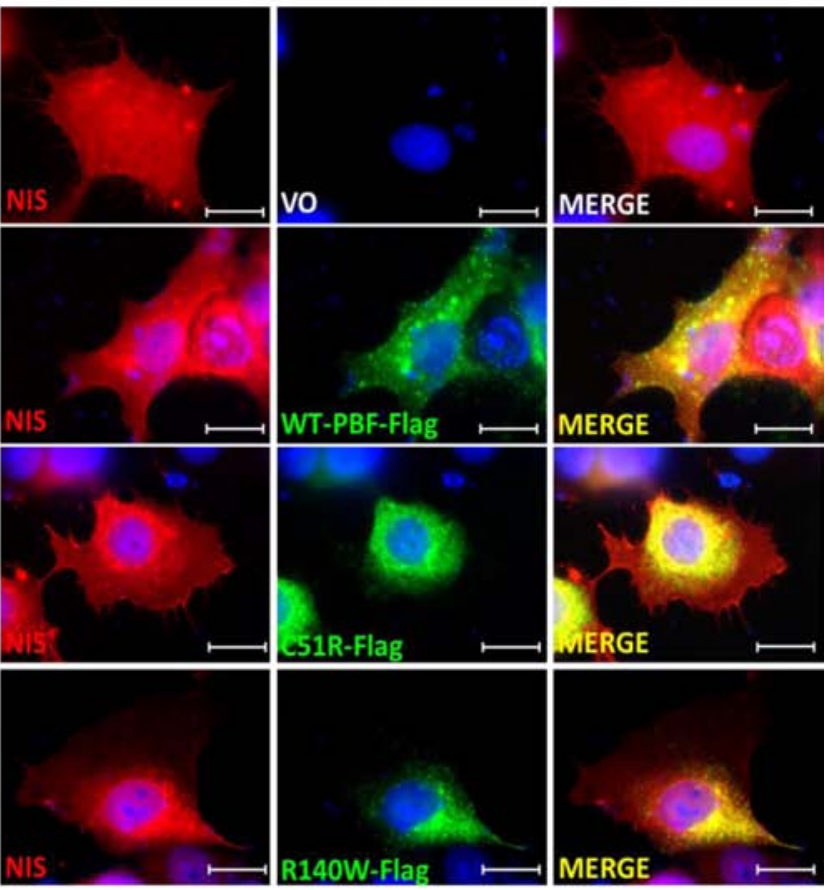

Figure 5

PBF mutations C51R and R140W retain the capability to repress radioiodide uptake in vitro. Radioiodide uptake experiments in TPC1 (A) and MCF7 (B) cells transiently transfected with VO, NIS-MYC and VO, WT PBF, C51R or R140W. Uptake was normalised to protein concentration and specific NISmediated uptake was demonstrated using the NIS inhibitor sodium perchlorate. Data presented as mean values \pm S.E. $(n=3)$. $* * P<0.01, * * * P<0.001$. Immunofluorescent microscopy of NIS-MYC (red) with FLAG-tagged WT PBF, C51R or R140W (green), using the NIS and FLAG antibodies, respectively, in HeLa (C) and MCF7 (D) cells. NIS-MYC was observed predominantly at the plasma membrane with VO co-transfection and with the co-transfection of WT PBF, C51R or R140W mislocalised to intracellular vesicles, ER and Golgi, respectively, where co-localisation was observed (yellow). Magnification $=100 \times$. Bars $=20 \mu \mathrm{m}$. 
may not be confined to the plasma membrane and intracellular vesicles.

\section{C51R and R140W substitutions lose cell transforming ability compared to WT PBF}

Overexpression of PBF induces cellular transformation in vitro (Stratford et al. 2005). To determine whether PBF mutants C51R and R140W retain this ability, we firstly utilised colony formation assays in TPC1 cells stably transfected with VO, WT PBF, C51R or R140W. In contrast to WT PBF, both mutants failed to induce colony formation (Fig. 6A). We further conducted soft agar experiments in NIH $3 \mathrm{~T} 3$ cells stably transfected with VO, WT PBF, C51R or R140W mutants. WT PBF demonstrated a dramatic increase in transforming ability compared to stable VO controls (Fig. 6B), as we have reported previously (Stratford et al. 2005). However, mutants C51R and R140W entirely lost the ability to elicit anchorageindependent growth in soft agar assays (Fig. 6B).

Taken together, the rare PBF mutations recently reported in multiple human tumour types are unlikely to be aetiological. Although some canonical PBF functionality is retained, we propose that PBF overexpression in human tumours, rather than sequence alteration, is the more

A
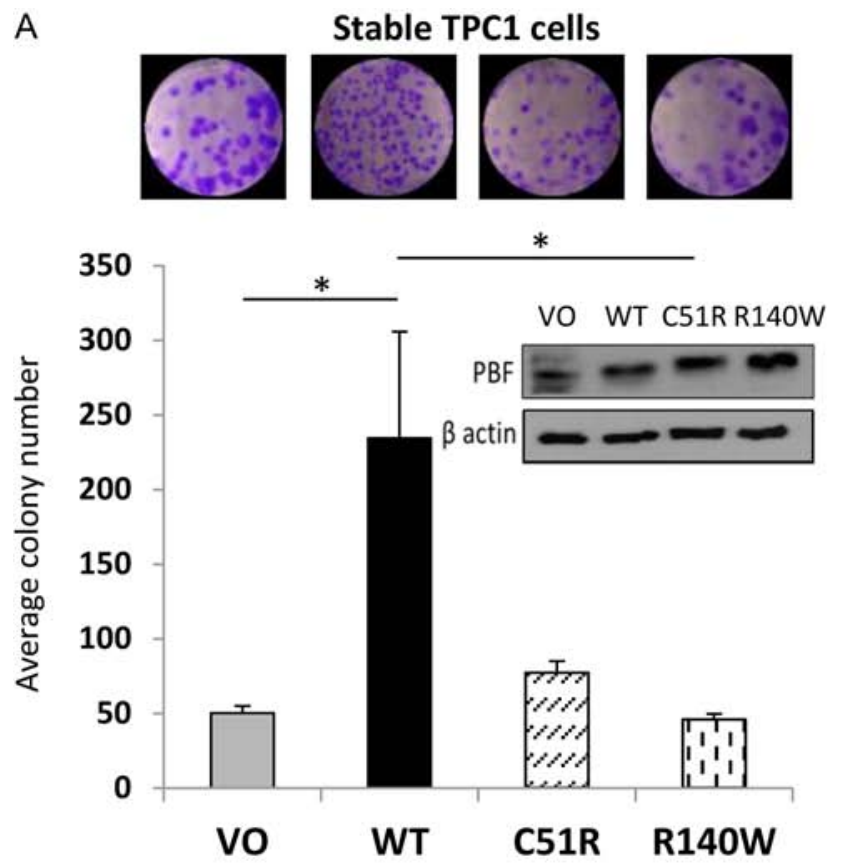

important driver of cellular transformation. Furthermore, these findings suggest that caution is required in the interpretation of whole-genome sequencing of tumours, given that even mutations in established oncogenes are frequently passenger events.

\section{Discussion}

Recent initiatives such as COSMIC and TCGA are unearthing a myriad of mutational events in thousands of human tumours. However, only a minority of these will confer selective growth advantage and clonal expansion of the cells in which they are found. The silent majority are likely to represent the product of the unstable genetic environments which generally develop during cancer progression.

A number of statistical approaches have been utilised to help predict driver mutations and genes, based largely on protein function, mutational frequency and pathway involvement. One such statistical approach - the Driver Oncogene and Tumor Suppressor Finder - predicted that the proto-oncogene PBF/PTTG1IP may be one of 12 genes driving thyroid carcinogenesis in 326 tumour samples from TCGA (Melloni et al. 2014). We have previously appraised the oncogenic function of PBF in numerous

B Stable NIH/3T3 cells
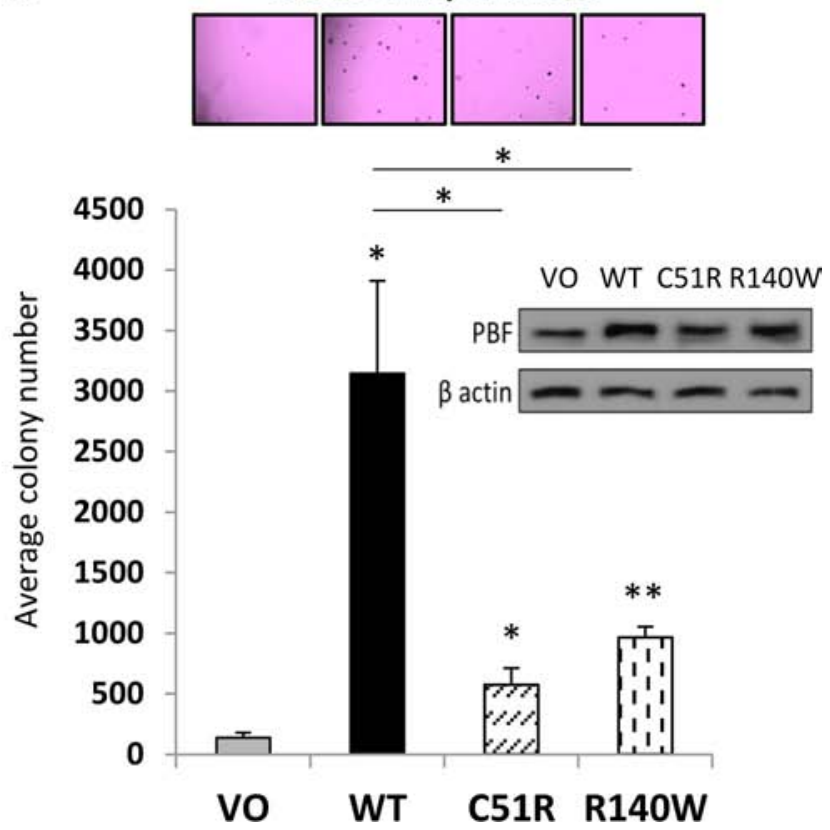

Figure 6

C51R and R140W substitutions lose cell transformation ability compared to WT PBF. (A) Colony formation assays in TPC1 cells stably transfected with VO, WT PBF, C51R and R140W; representative photomicrographs shown above. (B) Soft agar assays in NIH 3 T3 cells stably transfected with VO, WT PBF, C51R and R140W mutants. Representative images above illustrate relative numbers of colonies. Inset - Western blots of PBF expression in the stable cell lines using an anti-PBF antibody. Data presented as mean values \pm s.e. ( $n=3$, each with $>3$ replicates). ${ }^{*} P<0.05$.

http://erc.endocrinology-journals.org DOI: $10.1530 /$ ERC-16-0340
(C) 2017 The authors Printed in Great Britain
Published by Bioscientifica Ltd. 
studies, and we and others have reported significantly induced expression of the gene in thyroid, breast, pituitary and colorectal tumours (Boelaert et al. 2003, McCabe et al. 2003, Stratford et al. 2005, Watkins et al. 2010, Hsueh et al. 2013, Read et al. 2016). The possibility that mutations in PBF may be oncogenic led us to evaluate the functional effect of the first reported COSMIC mutations. Using PBF as a model gene to interrogate the findings of COSMIC and TCGA, we now report that even mutations in genes with known oncogenic functions do not necessarily result in growth advantage or other hallmarks of driver gene aetiology.

PBF is a widely expressed glycoprotein with a 180 amino acid sequence possessing an $\mathrm{N}$-terminal signal peptide, a transmembrane domain, a bipartite nuclear localisation signal and a tyrosine-sorting signal. PBF shares no homology with any other human proteins but is well conserved among a diverse range of animal species. Having established the basic biochemistry of the first 10 mutations reported in COSMIC, we narrowed our subsequent functional studies to two potentially key substitutions: C51R and R140W. One criticism of this approach is that we may have missed genuinely oncogenic activities in the 8 other mutations which we did not pursue in depth. However, it was impractical to perform, for example, simultaneous 2D invasion assays for all 10 mutations, and initial proliferation data suggested no significant alterations in cell turnover. Our initial thought was that residue G106 might represent a hot-spot of mutational activation. However, G106V/R/W all failed to significantly enhance markers of cell turnover, and their appearance in COSMIC may therefore be coincidental. Additionally, the G106R mutation was particularly unstable, decreasing its likelihood of sustained oncogenic activity.

Currently, there are 28 reported PBF mutations in the COSMIC database, the vast majority being single amino acid substitutions. The overall incidence of PBF mutations across all cancers sequenced to date in the TCGA project is less than $0.5 \%$. No PBF mutations have been reported in thyroid cancer; indeed the majority of mutations in this study were apparent in colorectal cancer. Additionally, there was no apparent correlation between PBF mutation status and clinical outcome in colorectal cancer. This is in contrast to previously published clinical associations in colorectal cancer, as well as in thyroid and breast neoplasia, where PBF expression significantly correlated with tumour grade and/or patient outcome (Boelaert et al. 2003, McCabe et al. 2003,
Stratford et al. 2005, Watkins et al. 2010, Hsueh et al. 2013, Read et al. 2016).

One interesting facet of our in vitro work was that mutations abrogated certain functions of PBF (cell invasion, migration, colony formation), whilst retaining others (proliferation, radioiodide uptake repression). This sheds new light particularly into the repression of NIS function, in that C51R (predominantly restricted to the ER) and R140W (mainly in the Golgi) still co-localised with NIS and markedly inhibited radioiodide uptake. PBF binds to NIS (Smith et al. 2009, 2013) and, while not demonstrating an interaction between NIS and these mutants, we would now speculate that NIS:PBF interaction can occur in multiple cellular compartments, and not solely at the plasma membrane and in intracellular vesicles as previously hypothesised (Smith et al. 2009). In stark contrast, C51R and R140W both lost the ability to induce cell invasion, migration, colony formation and anchorageindependent growth. We suggest that these processes are all dependent upon correct subcellular localisation of PBF, particularly as the protein stability of C51R and R140W was not significantly different from WT. C51R also showed altered glycosylation. Although we frequently detect - and have now confirmed - glycosylation and dimerisation of PBF, the functional significance of these post-translational processes is not currently known. It would thus appear that glycosylation, dimerisation and subcellular localisation differentially impact on PBF's range of in vitro functions, which we are currently trying to understand in more detail. One caveat here is that while we did not find that the tags used (HA, FLAG and MYC) impacted upon WT PBF processing, localisation or function, there remains the possibility that PBF mutants may be differentially 'sensitive' to the addition of tags. This would need to be assessed in further studies.

Importantly, in the standard biochemical assays employed here we were unable to detect any gain-offunction. Although C51R demonstrated increased cell turnover in BrdU assays, this did not translate to enhanced colony formation or anchorage-independent growth.

We therefore conclude that rare mutations in PBF are unlikely to be driver events in human cancer. Overexpression of WT PBF, which has been associated with tumour induction in xenograft models (Stratford et al. 2005), hyperplasia and follicular lesions in transgenic mice (Read et al. 2011), extramural vascular invasion in human colorectal tumours (Read et al. 2016), and reduced disease-specific survival in patients with thyroid tumours (Hsueh et al. 2013), is a more pertinent driver event. The 
TCGA data suggest that an increase in PBF expression could often be at least partly due to increased copy number (Fig. 1). However, the paucity of $P B F$ alterations in the thyroid cancer dataset fails to explain the high levels of PBF in these tumours and suggests the presence of other, as yet unknown, mechanisms that lead to increased PBF expression. Thus, why PBF is upregulated in tumours, and exactly how this induction drives hyperplastic and neoplastic progression, remains ill defined.

\section{Supplementary data}

This is linked to the online version of the paper at http://dx.doi.org/10.1530/ ERC-17-0340.

\section{Declaration of interest}

The authors declare that there is no conflict of interest that could be perceived as prejudicing the impartiality of the research reported.

\section{Funding}

This work was supported by the Faculty of Medicine Siriraj Hospital, Mahidol University, Bangkok, Thailand and the Medical Research Council (grant number RRAK16296).

\section{References}

Boelaert K, McCabe CJ, Tannahill LA, Gittoes NJ, Holder RL, Watkinson JC, Bradwell AR, Sheppard MC \& Franklyn JA 2003 Pituitary tumor transforming gene and fibroblast growth factor-2 expression: potential prognostic indicators in differentiated thyroid cancer. Journal of Clinical Endocrinology and Metabolism 88 2341-2347. (doi:10.1210/jc.2002-021113)

Boelaert K, Smith VE, Stratford AL, Kogai T, Tannahill LA, Watkinson JC, Eggo MC, Franklyn JA \& McCabe CJ 2007 PTTG and PBF repress the human sodium iodide symporter. Oncogene 26 4344-4356. (doi:10.1038/sj.onc.1210221)

Cerami E, Gao J, Dogrusoz U, Gross BE, Sumer SO, Aksoy BA, Jacobsen A, Byrne CJ, Heuer ML, Larsson E, et al. 2012 The cBio cancer genomics portal: an open platform for exploring multidimensional cancer genomics data. Cancer Discovery 2 401-404. (doi:10.1158/2159-8290. CD-12-0095)

Chin L, Andersen JN \& Futreal PA 2011 Cancer genomics: from discovery science to personalized medicine. Nature Medicine 17 297-303. (doi:10.1038/nm.2323)

Forbes SA, Bhamra G, Bamford S, Dawson E, Kok C, Clements J, Menzies A, Teague JW, Futreal PA \& Stratton MR 2008 The Catalogue of Somatic Mutations in Cancer (COSMIC). Current Protocols in Human Genetics Chapter 10 Unit 10 11. (doi:10.1002/0471142905. hg1011s57)

Forbes SA, Bindal N, Bamford S, Cole C, Kok CY, Beare D, Jia M, Shepherd R, Leung K, Menzies A, et al. 2011 COSMIC: mining complete cancer genomes in the Catalogue of Somatic Mutations in Cancer. Nucleic Acids Research 39 D945-D950. (doi:10.1093/nar/gkq929)

Forbes SA, Beare D, Gunasekaran P, Leung K, Bindal N, Boutselakis H, Ding M, Bamford S, Cole C, Ward S, et al. 2015 COSMIC: exploring the world's knowledge of somatic mutations in human cancer. Nucleic Acids Research 43 D805-D811. (doi:10.1093/nar/gku1075)
Gao J, Aksoy BA, Dogrusoz U, Dresdner G, Gross B, Sumer SO, Sun Y, Jacobsen A, Sinha R, Larsson E, et al. 2013 Integrative analysis of complex cancer genomics and clinical profiles using the cBioPortal. Science Signaling 6 pl1. (doi.10.1126/scisignal.2004088)

Greenman C, Stephens P, Smith R, Dalgliesh GL, Hunter C, Bignell G, Davies H, Teague J, Butler A, Stevens C, et al. 2007 Patterns of somatic mutation in human cancer genomes. Nature 446 153-158. (doi:10.1038/nature05610)

Hsueh C, Lin JD, Chang YS, Hsueh S, Chao TC, Yu JS, Jung SM, Tseng NM, Sun JH, Kuo SY, et al. 2013 Prognostic significance of pituitary tumour-transforming gene-binding factor (PBF) expression in papillary thyroid carcinoma. Clinical Endocrinology 78 303-309. (doi:10.1111/cen.12007)

McCabe CJ, Khaira JS, Boelaert K, Heaney AP, Tannahill LA, Hussain S, Mitchell R, Olliff J, Sheppard MC, Franklyn JA, et al. 2003 Expression of pituitary tumour transforming gene (PTTG) and fibroblast growth factor-2 (FGF-2) in human pituitary adenomas: relationships to clinical tumour behaviour. Clinical Endocrinology 58 141-150. (doi:10.1046/j.1365-2265.2003.01598.x)

Melloni GE, Ogier AG, de Pretis S, Mazzarella L, Pelizzola M, Pelicci PG \& Riva L 2014 DOTS-Finder: a comprehensive tool for assessing driver genes in cancer genomes. Genome Medicine 6 44. (doi:10.1186/ gm563)

Read ML, Lewy GD, Fong JC, Sharma N, Seed RI, Smith VE, Gentilin E, Warfield A, Eggo MC, Knauf JA, et al. 2011 Proto-oncogene PBF/ PTTG1IP regulates thyroid cell growth and represses radioiodide treatment. Cancer Research 71 6153-6164. (doi:10.1158/0008-5472. CAN-11-0720)

Read ML, Seed RI, Fong JC, Modasia B, Ryan GA, Watkins RJ, Gagliano T, Smith VE, Stratford AL, Kwan PK, et al. 2014 The PTTG1-binding factor (PBF/PTTG1IP) regulates p53 activity in thyroid cells. Endocrinology 155 1222-1234. (doi:10.1210/ en.2013-1646)

Read ML, Seed RI, Modasia B, Kwan PP, Sharma N, Smith VE, Watkins RJ, Bansal S, Gagliano T, Stratford AL, et al. 2016 The protooncogene PBF binds p53 and is associated with prognostic features in colorectal cancer. Molecular Carcinogenesis 55 15-26. (doi:10.1002/ mc.22254)

Sim NL, Kumar P, Hu J, Henikoff S, Schneider G \& Ng PC 2012 SIFT web server: predicting effects of amino acid substitutions on proteins. Nucleic Acids Research 40 W452-W457. (doi:10.1093/nar/ gks539)

Smith VE, Read ML, Turnell AS, Watkins RJ, Watkinson JC, Lewy GD, Fong JC, James SR, Eggo MC, Boelaert K, et al. 2009 A novel mechanism of sodium iodide symporter repression in differentiated thyroid cancer. Journal of Cell Science 122 3393-3402. (doi:10.1242/ jcs.045427)

Smith VE, Franklyn JA \& McCabe CJ 2011 Expression and function of the novel proto-oncogene PBF in thyroid cancer: a new target for augmenting radioiodine uptake. Journal of Endocrinology $\mathbf{2 1 0}$ 157-163. (doi:10.1530/JOE-11-0064)

Smith VE, Read ML, Turnell AS, Sharma N, Lewy GD, Fong JC, Seed RI, Kwan P, Ryan G, Mehanna H, et al. 2012 PTTG-binding factor (PBF) is a novel regulator of the thyroid hormone transporter MCT8. Endocrinology 153 3526-3536. (doi:10.1210/en.2011-2030)

Smith VE, Sharma N, Watkins RJ, Read ML, Ryan GA, Kwan PP, Martin A, Watkinson JC, Boelaert K, Franklyn JA, et al. 2013 Manipulation of PBF/PTTG1IP phosphorylation status; a potential new therapeutic strategy for improving radioiodine uptake in thyroid and other tumors. Journal of Clinical Endocrinology and Metabolism 98 2876-2886. (doi:10.1210/jc.2012-3640)

Stratford AL, Boelaert K, Tannahill LA, Kim DS, Warfield A, Eggo MC, Gittoes NJ, Young LS, Franklyn JA \& McCabe CJ 2005 Pituitary tumor transforming gene binding factor: a novel transforming gene in thyroid tumorigenesis. Journal of Clinical Endocrinology and Metabolism 90 4341-4349. (doi:10.1210/jc.2005-0523) 
Tomczak K, Czerwinska P \& Wiznerowicz M 2015 The Cancer Genome Atlas (TCGA): an immeasurable source of knowledge. Contemporary Oncology 19 A68-A77. (doi.10.5114/wo.2014.47136)

Vlotides G, Eigler T \& Melmed S 2007 Pituitary tumor-transforming gene: physiology and implications for tumorigenesis. Endocrine Reviews 28 165-186. (doi:10.1210/er.2006-0042)

Watkins RJ, Read ML, Smith VE, Sharma N, Reynolds GM, Buckley L, Doig C, Campbell MJ, Lewy G, Eggo MC, et al. 2010 Pituitary tumor transforming gene binding factor: a new gene in breast cancer. Cancer Research 70 3739-3749. (doi:10.1158/0008-5472.CAN-09-3531)
Watkins RJ, Imruetaicharoenchoke W, Read ML, Sharma N, Poole VL, Gentilin E, Bansal S, Bosseboeuf E, Fletcher R, Nieto HR, et al. 2016 Pro-invasive effect of proto-oncogene PBF is modulated by an interaction with cortactin. Journal of Clinical Endocrinology and Metabolism 101 4551-4563. (doi:10.1210/jc.2016-1932)

Xiang C, Gao H, Meng L, Qin Z, Ma R, Liu Y, Jiang Y, Dang C, Jin L, He F, et al. 2012 Functional variable number of tandem repeats variation in the promoter of proto-oncogene PTTG1IP is associated with risk of estrogen receptor-positive breast cancer. Cancer Science 103 1121-1128. (doi:10.1111/j.1349-7006.2012.02266.x)

Received in final form 28 June 2017

Accepted 3 July 2017

Accepted Preprint published online 4 July 2017 